\title{
Ultrasound elastography can detect placental tissue abnormalities
}

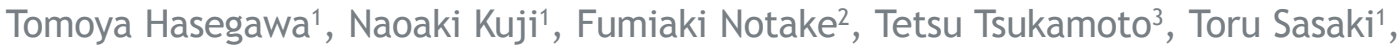 \\ Motohiro Shimizu ${ }^{4}$, Kazunori Mukaida ${ }^{1}$, Hiroe Ito ${ }^{1}$, Keiichi Isaka ${ }^{1}$, Hirotaka Nishi ${ }^{1}$

\footnotetext{
${ }^{1}$ Department of Obstetrics and Gynecology, Tokyo Medical University, Tokyo, Japan

${ }^{2}$ Radiation Division, Tokyo Medical University Hachioji Medical Center, Tokyo, Japan

3 Diagnostic Pathology Division, Tokyo Medical University Hachioji Medical Center, Tokyo, Japan
} \\ ${ }^{4}$ Obstetrics and Gynecology, Tokyo Medical University Hachioji Medical Center, Tokyo, Japan
}

Radiol Oncol 2018; 52(2): 129-135.

Received 20 November 2017

Accepted 23 April 2018

Correspondence to: Tomoya Hasegawa, Department of Obstetrics and Gynecology, Tokyo Medical University, 6-7-1, Nishi-Shinjyuku, Shinjyuku, Tokyo, 160-0023, Japan. Phone: +8133342 6111; Fax: +8133342 6112; E-mail: ppppq999@gmail.com

Disclosure: No potential conflicts of interest were disclosed.

Background. In this prospective cohort study, we examined the utility of elastography to evaluate the fetus and placenta.

Patients and methods. Pregnant women in their third trimester of pregnancy, by which time the placenta has formed, were included in this study. A total of 111 women underwent ultrasound examinations, including elastography. Elastographic evaluation was performed using two protocols. First, the placental index (PI) was measured, which quantitatively assesses the hardness of tissue. Second, regions of interest (ROI) were categorized into 3-step scores according to the frequency of the blue area (hardness of placental tissue score [HT score]), which is a qualitative method. After delivery, 40 of the 111 placentas were pathologically examined.

Results. The average PI was $44.3( \pm 29.4)$ in the in utero SGA group, which was significantly higher than that in the normal group (8.8 ( \pm 10.0); $p<0.01$ ) during pregnancy. There was a significant correlation between the Pl and z score for estimated fetal weight (EFW) $(r=-0.55 ; p<0.01)$. Moreover, a significant positive correlation was observed between the Pl and the $z$ score of birth weight $(r=-0.39 ; p<0.01)$. Pathological ischemia findings of the placenta were identified in $67 \%$ of the HT score 3 group, representing 6 of the 9 patients, and in $20 \%$ of the HT score 1 group, representing only 3 of the 15 patients.

Conclusions. Placental hardness, as determined by elastography, correlates with both lower estimated fetal body weight and birth weight. These results suggest that ultrasound elastography in the placenta may be an additional marker of intrauterine fetal well-being.

Key words: elastography; placenta; small for gestational age; ultrasound

\section{Introduction}

When ultrasound elastography was first clinically applied for the evaluation of disease, it was used to differentiate cancer tissue from surrounding normal tissue according to stiffness. ${ }^{1-3}$ Since the elasticity of tissues and organs can be evaluated objectively, even in inaccessible parts of the living body ${ }^{4,5}$, this modality has been applied in the clinical diagnosis of breast cancer. ${ }^{6}$
Recently, elastography has been found to be useful for the evaluation of pathological changes in noncancerous tissues. For example, the stiffness of liver tissues is considered to relatively increase with an increase in the density of vessels. In particular, the severity of hepatic fibrosis, as determined by biopsy, actually showed a correlation with a color score found using elastography in the liver area., 8

Small neonates who did not reach an appropriate weight according to gestational age in weeks 
are referred to as small for gestational age (SGA). Perinatal mortality and the incidence of mental deficiency are high for SGA infants compared with non-SGA infants. Therefore, a fetus with SGA represents a high-risk pregnancy. ${ }^{9}$ Screening for SGA in the uterus is important during prenatal exami-
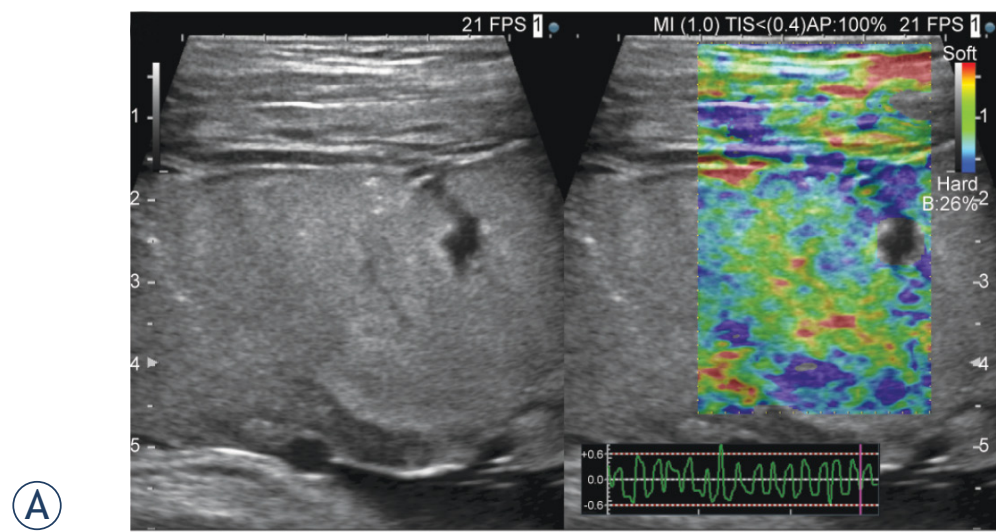

(B)

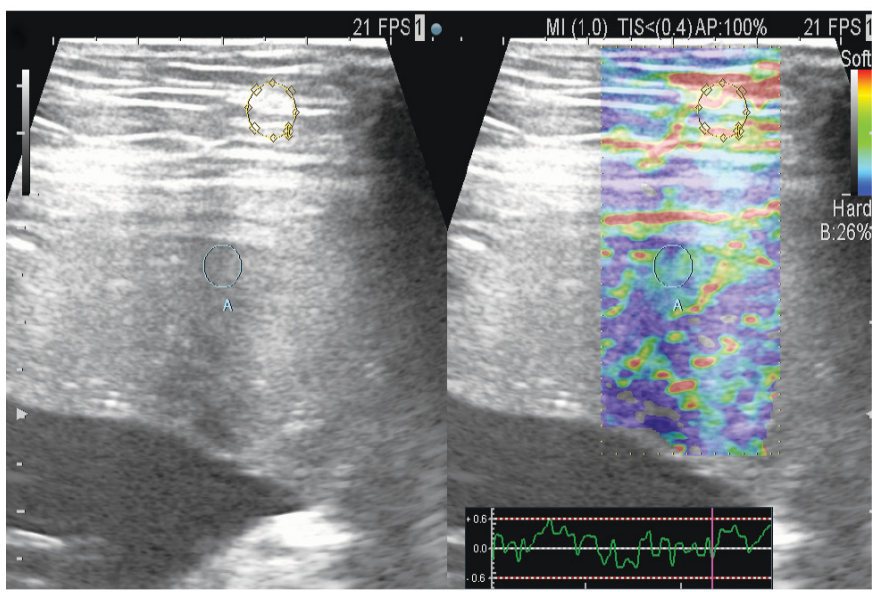

\section{(C)}

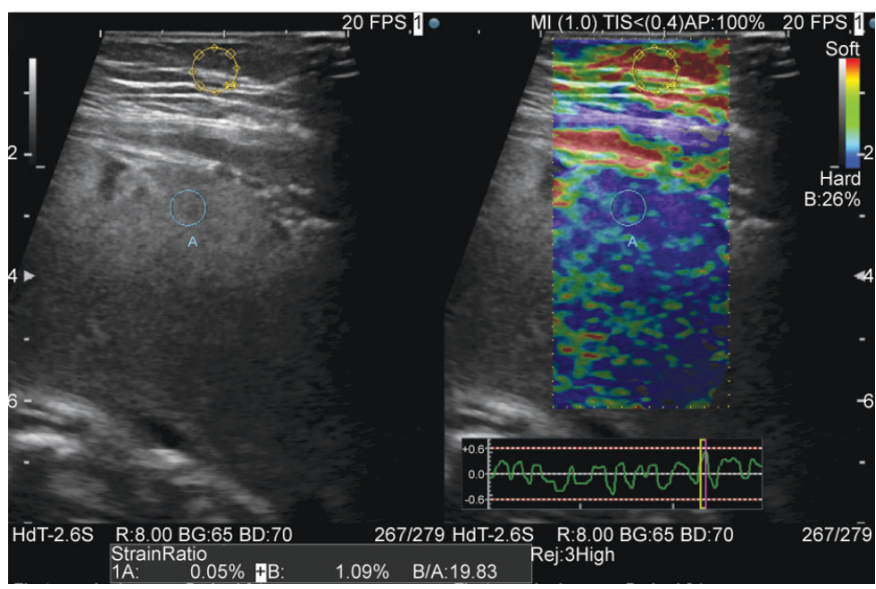

FIGURE 1. Representative ultrasound images of hardness of tissue (HT) score (A) The HT score was 1 when the blue area in the ROI was $50 \%$ or less. A strain graph, used to assess proper application of compression, is indicated in the lower right (B) The blue and green areas are shown mixed together in relatively equal percentages in the placenta (C) Most of the placenta is indicated in blue. In the left image, the strain ratio is calculated from the region of interest of the fat and placental tissue. In this case, the placental index is 19.83 . nations; however, pathological conditions vary, and examination methods and indices for discrimination are not sufficient. ${ }^{10,11}$

Ultrasonography has long been a valuable tool for the diagnosis of pathological conditions during pregnancy when the use of diagnostic radiation imaging or invasive examination is avoided. The ultrasound frequency commonly used for elastography is equivalent to that of B mode, and it is thought that there is little effect on fetuses during pregnancy. ${ }^{12}$ Among the few reports of elastography used in obstetrics, some have evaluated the cervix and its correlation with premature labor. ${ }^{13-15}$ Therefore, in this study, we investigated the correlation between the elasticity of placental tissue, as evaluated by ultrasound elastography, and SGA.

\section{Materials and Patiens}

A prospective cohort study was performed on a total of 111 pregnant women who regularly visit our hospital received examinations. Only cases with anterior-lying placenta were included to avoid the effect of amniotic fluid. Only singleton pregnancies were included. For both fetal biometry and strain elastography, Ascendus (Hitachi Aloka Medical) with Eup-L52 probes were used for ultrasound examinations.

After the regular prenatal examination was performed (biparietal diameter, abdominal circumference, and femur length of the fetus), elastographic evaluation was performed using two protocols. First, the fat lesion ratio (FLR), which expresses the ratio of the elasticity of the target tissues (placenta) to subcutaneous fat (reference), was measured and calculated according to the manufacturer's instructions and defined as the placental index (PI). According to the diagnostic modality used for mammary tissue, two regions of interest (ROI) with 2-cm diameters were randomly set for the fat and placenta for comparison. Second, we made a benchmark to easily determine the hardness of the placenta, and the color mapping patterns of certain ROIs of the placenta were evaluated by an observer. The pattern was categorized into 3-step scores according to the frequency of the blue area (hardness of placental tissue score [HT score]). The HT score was 1 when the blue area in the ROI was $<50 \%$, 2 when the blue area was between $50 \%$ and $75 \%$, and 3 when the blue area was $>75 \%$ (Figure 1 ).

The HT score was evaluated by an obstetrician who was blinded to fetal biometry and perinatal information. Other blinded physician was asked to 
evaluate the score, and a consensus between two examiners was adopted as the HT score for the placenta.

We took measurements by avoiding large blood vessels and thereby avoided bias caused by areas without echo. The ROI was selected within $10 \mathrm{~cm}$ from the body surface; areas without tissue elasticity, such as the deep part, were avoided. Also, an attached strain graph was used to confirm whether adequate pressure was applied ${ }^{16}$ (Figure 1A, lower right).

The evaluation of reliability in elastography measurements was conducted using intra-class correlation coefficients (ICC). The intra-tester ICC was calculated for the 3 measurements in each placenta.

After delivery, the placentae of 40 patients who agreed to undergo a pathological test were investigated. Pathological examination was done blindly by a pathologist who was unaware about the patient's obstetric information.

Statistical testing was performed using Welch's $\mathrm{t}$-test for patient characteristics and a $\mathrm{z}$ score of the estimated fetal weight (EFW) (significance level, $0.05 \%$ ). Pearson's rank-correlation coefficient was calculated to assess the correlation between the PI and pregnancy outcomes. A multivariate linear regression analysis was performed with the EFW z score as a dependent variable and age, parity, placental weight at delivery, PI, and HT score as independent variables. SPSS version 24.0 was used for all statistical computations.

All procedures performed in studies involving human participants were in accordance with the ethical standards of the institutional and/or national research committee and with the 1964 Helsinki declaration and its later amendments or comparable ethical standards. This study was approved by the Institutional Review Board of the Tokyo Medical University (No. 2949). Informed consent was obtained from all patients before they were enrolled in the study.

\section{Results}

The patient characteristics of the normal EFW group and the in utero SGA group are shown in Table 1. Five pregnant women from normal groups were randomly selected to evaluate the reliability in elastography measurements. The intra-tester ICC was 0.987 , thereby indicating a high reliability.

An EFW with a standard deviation (SD) of -1.5 or lower was defined as SGA. ${ }^{17}$ The average PI was
TABLE 1. Population features between the Normal group and SGA group

\begin{tabular}{lccc} 
& Normal group & SGA group & $P$ \\
\hline Number & 101 & 10 & \\
Maternal age (years) & $34.4( \pm 5.6)$ & $33.6( \pm 4.7)$ & NS \\
Gravidity & $0.9( \pm 1.2)$ & $1.3( \pm 1.3)$ & NS \\
Parity & $0.5( \pm 0.9)$ & $0.6( \pm 0.7)$ & NS \\
Gestational age (weeks) & $31.7( \pm 6.3)$ & $32.8( \pm 4.2)$ & NS \\
EFW (SD) & $+0.11( \pm 0.74)$ & $-2.33( \pm 0.84)$ & $P<0.01$ \\
Placental Index & $8.8( \pm 10.0)$ & $44.3( \pm 29.4)$ & $P<0.01$ \\
HT score & $1.56( \pm 0.68)$ & $2.70( \pm 0.67)$ & $P<0.01$ \\
Birth weight (SD) & $0.11( \pm 1.13)$ & $-2.19( \pm 1.07)$ & $P<0.01$ \\
Placenta weight $(g)$ & $562.3( \pm 119.7)$ & $366( \pm 120.0)$ & $P<0.01$ \\
\hline
\end{tabular}

The above values represent the mean (standard deviation).

$\mathrm{EFW}=$ estimated fetal weight; $\mathrm{HT}=$ score hardness of tissue score; SGA = small for gestational age

TABLE 2. HT score and pathological findings related to placental ischemia after delivery

\begin{tabular}{lccc}
\hline HT score & $\begin{array}{c}\mathbf{1} \\
(\mathrm{n}=\mathbf{1 5})\end{array}$ & $\begin{array}{c}\mathbf{2} \\
(\mathrm{n}=16)\end{array}$ & $\begin{array}{c}\mathbf{3} \\
(\mathrm{n}=\mathbf{9})\end{array}$ \\
\hline Accelerated maturation of villi $(+),(\mathrm{n})$ & 1 & 2 & 2 \\
Infarction $(+),(\mathrm{n})$ & 2 & 1 & 3 \\
Villous inflammation $(+),(\mathrm{n})$ & 0 & 1 & 1 \\
Total, $\mathrm{n},(\%)$ & $3(20)$ & $4(25)$ & $6(67)$ \\
\hline
\end{tabular}

HT scores were evaluated during elastography. The presence and absence of pathological findings of the placenta after delivery are shown.

8.8 in the normal group and 44.3 in the in utero SGA group. The in utero SGA group had a higher placenta hardness score than did the normal group.

In this study, significant differences were not observed for the prognosis of cesarean section rate, hemorrhage volume, and Apgar score of the child at delivery during the perinatal period.

The correlation between the PI and z score for EFW is shown in Figure 2. There was a significant correlation between the PI and z score for EFW (correlation coefficient, $r=-0.55 ; p<0.01$ ). Moreover, a significant positive correlation was observed between the PI and the $\mathrm{z}$ score of birth weight (correlation coefficient, $r=-0.39 ; p<0.01$ ) (Figure 3). In the multivariate analysis, HT score and PI were significantly associated with EFW $(\beta=-0.398, p=0.01$; and $\beta=0.425, p=0.007$, respectively). There was no statistically significant association between the other parameters and EFW. Table 2 shows a correlation between pathological findings of the placenta after delivery and HT score. After delivery, the 


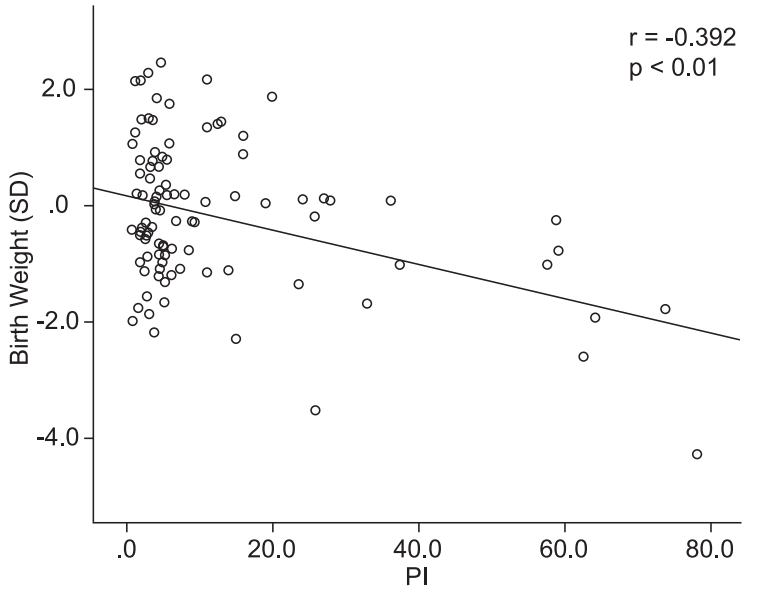

FIGURE 2. The correlation between the placental index (PI) and $z$ score for estimated fetal weight at prenatal examination (EFW). There is a significant correlation between the $\mathrm{PI}$ and $\mathrm{z}$ score for EFW (correlation coefficient, $r=-0.55 ; p<0.01$ ).

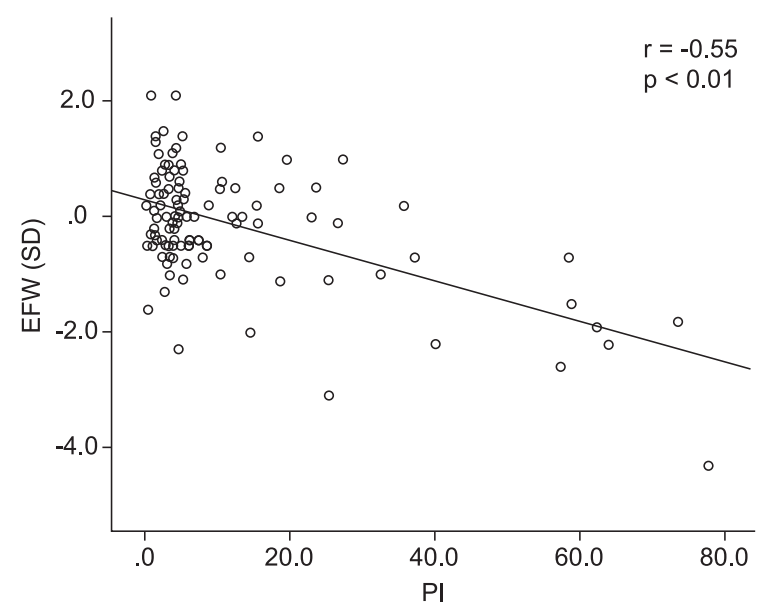

FIGURE 3. A significant positive correlation was observed between the placental index (PI) and z score of birth weight (correlation coefficient, $r=-0.39 ; p<0.01$ ).

pathologist checked for three types of accelerated maturation of villi, which reflect vessels that have been reactively increased by ischemia, infarction, and villous inflammation. No significant differences were observed among all HT score groups, but the group with an HT score of 3 tended to have more findings related to ischemia of the placenta.

For the groups with HT scores of 1, 2, and 3, the mean birth weights were -0.11 SD, -0.09 SD, and -0.93 SD, respectively. The differences between HT scores 1 and 2 and between HT scores 2 and 3 were $p=0.067$ and $p=0.054$, respectively. Furthermore, when the results of elastography were compared between before and after the 30th week in the same cases, which allowed us to assess the effect of measurement timing on the HT score, no changes in HT score were observed in the 5 cases that received an elastography examination, except for 1 case.

No obvious maternal or fetal disadvantages, such as premature birth or abruption, developed after the elastography examination.

\section{Discussion}

There have been several reports describing the use of elastography to examine the placenta. Recently, Cimsit et al. performed elastography by using shear wave imaging; they demonstrated that a placenta affected by pregnancy-induced hypertension was harder than a normal placenta. ${ }^{18}$

Using strain elastography with acoustic radiation (ARFI), Sugitani et al. discovered that the placenta of fetuses with fetal growth restriction (FGR) seemed harder than normal. They used elastography to study the placenta after delivery and measured the stiffness of the placenta with infarction. In doing so, they found that elastography was able to diagnose fibrogenesis without inflammation and congestion. ${ }^{19,20}$ However, their reports compared the elasticity of placentas from patients with pregnancy-induced hypertension with or without FGR. Furthermore, the safety of shear wave imaging with ARFI in the developing fetus has not been fully established because of the high energy of this modality. $^{21}$

SGA is associated with significant perinatal morbidity and mortality. ${ }^{22,23}$ Although uterine artery Doppler is clinically applied to predict FGR of SGA, its sensitivity, and predictive value are not satisfactory. ${ }^{24}$ Recently, using a rat model, Quibel et al. demonstrated that placentas from fetuses with experimental intrauterine growth restriction demonstrated greater stiffness. ${ }^{25}$ However, to our knowledge, no report has analyzed the correlation between elasticity of the placenta and SGA in humans in utero. For all of these reasons, we first assumed that the placental hardness correlates with SGA. As seen in Table 1, the backgrounds of the in utero SGA group were consistent with those in past reports about FGR.

EFW, birth weight, and placental weight in the in utero SGA group were significantly lower than those in the normal group. Furthermore, the PI and HT score in the in utero SGA group were significantly higher than those in the normal group in this study. This result suggests that the PI and HT score may be new evaluation items for SGA. 
In this study, we first examined PI, which is an objective and quantitative evaluation scale built into the mode used in elastography. The ratio of the elasticity of the abdominal wall fat tissue, which is considered to have the almost constant elasticity to the target tissue, was quantified by a pre-defined algorithm. This approach has been used instead of invasive biopsy in the mammary gland region to enable the diagnosis of mammary gland diseases. ${ }^{26}$ Statistically significant correlations were found between the PI and z scores of EFW and between the PI and z scores of birth-weight. Furthermore, the multivariate analysis revealed that the HT score and PI were associated with EFW. These results suggest that placental stiffness has a strong relationship with SGA, regardless of the measurement procedure.

The utility of elastography and color mapping has been established in regions such as the mammary gland, prostate gland, and thyroid., ${ }^{2,3}$ Furthermore, color mapping is valuable because it is an intuitive evaluation method. It allows visual determination, even in models without a particular algorithm. Moreover, obstetricians want to confirm the situation of the fetus as efficiently as possible in a short period. Therefore, we secondly developed a novel color mapping score to evaluate the elasticity of the placenta, which we termed the HT score. This score may be a simple screening tool that can be used to identify abnormalities in the placenta.

The HT score failed to show a correlation with the $\mathrm{z}$ score for birth weight. Although some additional factors during pregnancy after an HT score examination exist and affect birth weight, it may be possible that a significant difference could be confirmed in a series of cases. Moreover, this study result suggests that HT score does not change based on gestational weeks. Fortunately, it may be convenient to evaluate the stiffness of the placenta using the HT score during pregnancy.

Pathologic examination of placentae of those with FGR revealed fibrin deposits in the intervillous space. ${ }^{27}$ Also, there have been reports of significant pathological findings of infarction and villous inflammation in the placentas of infants with SGA. ${ }^{28}$ Therefore, in at least some cases of FGR, hypercoagulability in the placenta will result in blood clots and infarction, leading to a decrease in uteroplacental circulation, which contributes to FGR. As shown in Figures 4 and 5, white infarcts were macroscopically observed in parts of the placenta, and complete and incomplete infarction images of the villus were histopathologically observed in the placenta of FGR neonates with HT scores of 3.

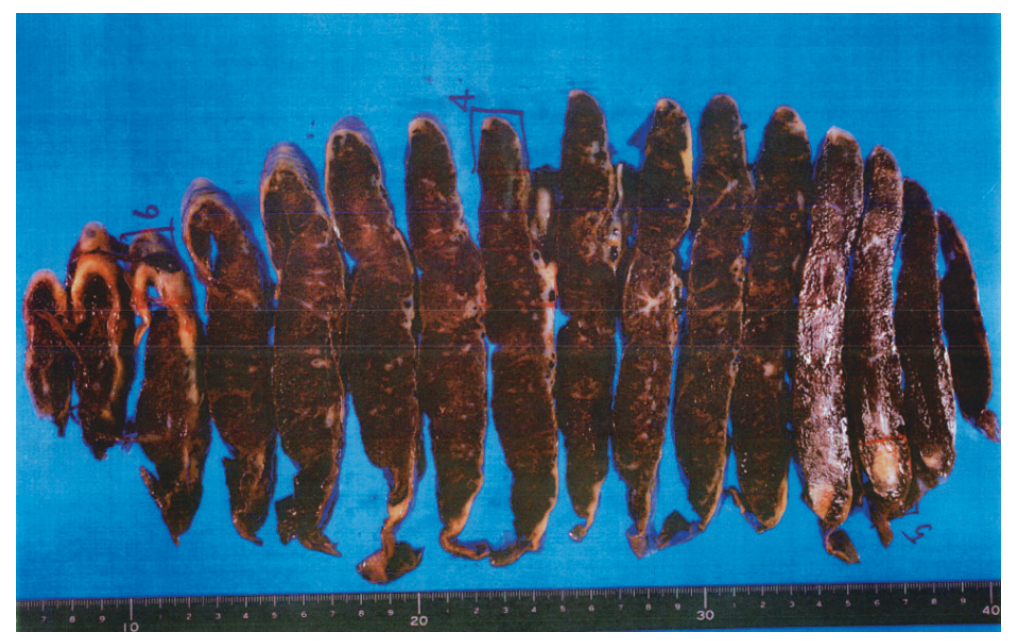

FIGURE 4. Gross pathological findings of the placenta. A macro-photograph of the placenta after delivery. The neonate had fetal growth restriction, diagnosed during elastography evaluation at week 28 of pregnancy. White placental infarction is observed at the bottom right of the photo.

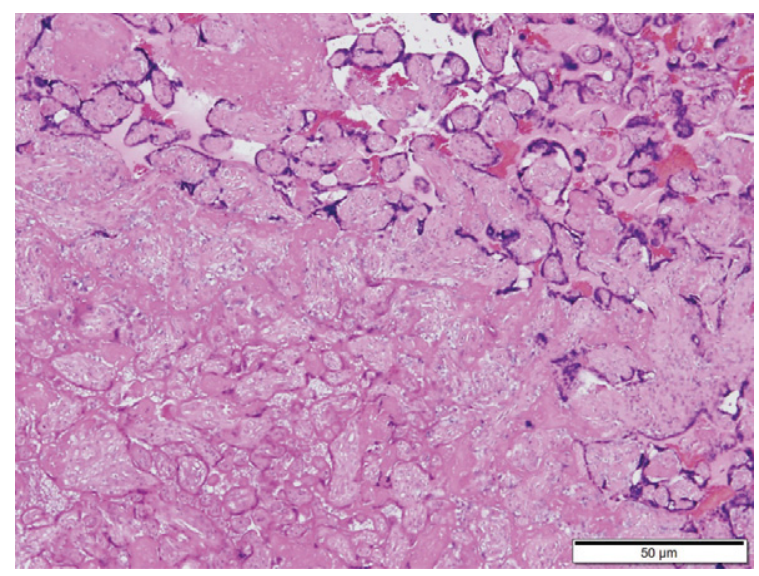

FIGURE 5. Histopathological findings of the placenta. Objective lens with $4 \times$ magnification. A micro-photograph of the same case as that shown in Figure 4. Complete infarction and incomplete infarction with remaining nuclear stainability are observed. The outlines of the villus remain in the upper right area.

Regarding the liver region, it has been reported that the pathological degree of fibrogenesis caused by chronic hepatitis and hepatic cirrhosis, and the hardness evaluated by strain elastography, are well correlated..$^{8,29}$ Our findings suggested that the harder the placenta, the more the infarction and villous inflammation tended to be. Thus, elastography may reflect their fibrogenesis of villi resulting from infarction, villous inflammation and insufficient oxygen supply of the placenta in SGA.

However, not all SGAs are pathologically growth restricted but may be constitutionally 
small for various other reasons, including genetic causes. Sequential EFW measurements of indices of fetal well-being (e.g., umbilical, middle cerebral, and uterine artery Doppler measurement; amniotic fluid volume, and fetal heart rate monitoring) may facilitate the identification of SGA due to placental insufficiency. Indeed, Karaman et al. described a putative association between the uterine artery pulsatility index and elastographic indices of the placenta. $^{30}$

Another limitation of this study is that strain elastography requires manual compression with the examiner's hands, although it has been reported that reproducible data can be obtained when a single examiner conducts the examination. ${ }^{31}$ Furthermore, only placentas located on the anterior uterine wall could be analyzed by our proto$\mathrm{col}$, since manual compression is nearly impossible for a posteriorly located placenta. Our study also exhibited notably variation in PI values according to measurement locations in the normal and SGA groups. Thus, further studies with standardized elastographic measurements are required to evaluate the condition of the entire placenta.

Despite these limitations, elastography may indicate some characteristics of the placenta. Through the technical development of devices, improvements in evaluation methods, algorithms, and/or combined use with other obstetric examination methods, elastography can become a new evaluation method for the fetus and placenta. ${ }^{18-20}$

In conclusion, we demonstrate that placental hardness during pregnancy correlates with low birth weight, based upon an assessment of the placenta using a non-invasive approach. Furthermore, hard placentas, as determined by elastography, may be indicative of pathological changes to the placenta in SGA.

\section{References}

1. Xing P, Wu L, Zhang C, Li S, Liu C, Wu C. Differentiation of benign from malignant thyroid lesions. J Ultrasound Med 2011; 30: 663-9. doi:10.7863/ jum.2011.30.5.663

2. Itoh A, Ueno E, Tohno E, Kamma H, Takahashi H, Shiina T, et al. Breast disease: Clinical application of US elastography for diagnosis. Radiology 2006; 239: 341-350. doi:10.1148/radiol.2391041676

3. Miyanaga N, Akaza H, Yamakawa M, Oikawa T, Sekido N, Hinotsu S, et al. Tissue elasticity imaging for diagnosis of prostate cancer: a preliminary report. Int J Urol 2006; 13: 1514-8. doi:10.1111/j.1442-2042.2006.01612.x

4. Ophir J, Cespedes I, Ponnekanti H, Yazdi Y, Li X. Elastography: a quantitative method for imaging the elasticity of biological tissues. Ultrason Imaging 1991; 13: 111-34. doi:10.1016/0161-7346(91)90079-W
5. Eggeb $\varnothing$ TM, $\varnothing$ kland I, Heien C, Gjessing LK, Romundstad P, Salvesen KÅ. Can ultrasound measurements replace digitally assessed elements of the Bishop score? Acta Obstet Gynecol Scand 2009; 88: 325-31. doi:10.1080/00016340902730417

6. Ueno E, Tohno E, Soeda S, Asaoka Y, Itoh K, Bamber JC, et al. Dynamic tests in real-time breast echography. Ultrasound Med Biol 1988; 14: 53-7.

7. Tatsumi $\mathrm{C}$, Kudo $\mathrm{M}$, Ueshima $\mathrm{K}$, Kitai $\mathrm{S}$, Takahashi $\mathrm{S}$, Inoue $\mathrm{T}$, et al. Noninvasive Evaluation of Hepatic Fibrosis Using Serum Fibrotic Markers, Transient Elastography (FibroScan) and Real-Time Tissue Elastography. Intervirology 2008; 51: 27-33. doi:10.1159/000122602

8. Tatsumi C, Kudo M, Ueshima K, Kitai S, Ishikawa E, Yada N, et al. Non-invasive evaluation of hepatic fibrosis for type C chronic hepatitis. Intervirology 2010; 53: 76-81. doi:10.1159/000252789

9. McIntire DD, Bloom SL, Casey BM, Leveno KJ. Birth weight in relation to morbidity and mortality among newborn infants. N Engl J Med 1999; 340: 1234-8. doi:10.1056/NEJM199904223401603

10. Pearce JM, Campbell S. A comparison of symphysis-fundal height and ultrasound as screening tests for light-for-gestational age infants. Br J Obstet Gynaecol 1987; 94: 100-4.

11. Duff GB. A randomized controlled trial in a hospital population of ultrasound measurement screening for the small for dates baby. Aust New Zeal J Obstet Gynaecol 1993; 33: 374-8. doi:10.1111/j.1479-828X.1993.tb02113.x

12. Makoto Yamakawa, Naotaka Nitta, Tsuyoshi Shiina, Takeshi Matsumura, Satoshi Tamano, Tsuyoshi Mitake, et al. High-speed Freehand Tissue Elasticity Imaging for Breast Diagnosis. Jpn J Appl Phys 2003; 42: 3265. doi:10.1143/JJAP.42.3265

13. Wozniak S, Czuczwar P, Szkodziak P, Milart P, Wozniakowska E, Paszkowski T. Elastography in predicting preterm delivery in asymptomatic, low-risk women: a prospective observational study. BMC Pregnancy Childbirth 2014; 14: 238. doi:10.1186/1471-2393-14-238

14. Hernandez-Andrade E, Hassan SS, Ahn H, Korzeniewski SJ, Leo $Y_{\text {, }}$ Chaiworapongsa $T$, et al. Evaluation of cervical stiffness during pregnancy using semiquantitative ultrasound elastography. Ultrasound Obstet Gynecol 2013; 41: 152-61. doi:10.1002/uog.12344

15. Swiatkowska-Freund M, Traczyk-Łoś A, Preis K, Łukaszuk M, Zielińska K. Prognostic value of elastography in predicting premature delivery. Ginekol Pol 2014; 85: 204-7.

16. Shiina T. JSUM ultrasound elastography practice guidelines: basics and terminology. J Med Ultrason 2013; 40: 309-23. doi:10.1007/s10396-0130490-z

17. Shinozuka N, Akamatsu N, Sato $\mathrm{S}$, Kanzaki T, Takeuch $\mathrm{H}$, Natori $\mathrm{M}$, et al. Ellipse tracing fetal growth assessment using abdominal circumference: JSUM standardization committee for fetal measurements. J Med Ultrasound 2000; 8: 87-94.

18. Cimsit C, Yoldemir T, Akpinar IN. Shear wave elastography in placental dysfunction. J Ultrasound Med 2015; 34: 151-9. doi:10.7863/ultra.34.1.151

19. Arena U, Vizzutti F, Corti G, Ambu S, Stasi C, Bresci S, et al. Acute vira hepatitis increases liver stiffness values measured by transient elastography. Hepatology 2007; 47: 380-4. doi:10.1002/hep.22007

20. Colli A, Pozzoni P, Berzuini A, Gerosa A, Canovi C, Molteni EE, et al. Decompensated chronic heart failure: Increased liver stiffness measured by means of transient elastography. Radiology 2010; 257: 872-8. doi:10.1148/ radiol.10100013

21. Sugitani M, Fujita Y, Yumoto $Y$, Fukushima K, Takeuchi T, Shimokawa M, et al. A new method for measurement of placental elasticity: Acoustic radiation force impulse imaging. Placenta 2013; 34: 1009-13. doi:10.1016/j. placenta.2013.08.014

22. Aucott SW, Donohue PK, Northington FJ. Increased morbidity in severe early intrauterine growth restriction. J Perinatol 2004; 24: 435-40. doi:10.1038/ sj.jp.7211116

23. Jacobsson B, Ahlin K, Francis A, Hagberg G, Hagberg H, Gardosi J. Cerebral palsy and restricted growth status at birth: population-based case-control study. BJOG An Int J Obstet Gynaecol 2008; 115: 1250-5. doi:10.1111/ j.1471-0528.2008.01827.x 
24. Cnossen JS, Morris RK, ter Riet G, Mol BWJ, van der Post JAM, Coomarasamy A, et al. Use of uterine artery Doppler ultrasonography to predict preeclampsia and intrauterine growth restriction: a systematic review and bivariable meta-analysis. Can Med Assoc J 2008; 178: 701-11. doi:10.1503/ cmaj.070430

25. Quibel T, Deloison B, Chammings F, Chalouhi GE, Siauve N, Aliison M, et al. Placental elastography in a murine intrauterine growth restriction model. Prenat Diagn 2015; 35: 1106-11. doi:10.1002/pd.4654

26. Ikeda K, Ogawa $Y$, Takii $M$, Sugano $K$, Ikeya T, Tokunaga $S$, et al. A role for elastography in the diagnosis of breast lesions by measuring the maximum fat lesion ratio (max-FLR) by tissue Doppler imaging. Breast Cancer 2012; 19: 71-6. doi:10.1007/s12282-011-0274-5

27. Sebire NJ, Backos M, Goldin RD, Regan L. Placental massive perivillous fibrin deposition associated with antiphospholipid antibody syndrome. BJOG 2002; 109: 570-3.

28. Maulik D, Frances Evans J, Ragolia L. Fetal growth restriction: pathogenic mechanisms. Clin Obstet Gynecol 2006; 49: 219-27.

29. Friedrich-Rust M, Ong M-F, Herrmann E, Dries V, Samaras $P$, Zeuzem S, et al. Real-time elastography for noninvasive assessment of liver fibrosis in chronic viral hepatitis. Am J Roentgenol 2007; 188: 758-64. doi:10.2214/ AJR.06.0322

30. Karaman E, Arslan H, Çetin O, Şahin HG, Bora A, Yavuz A, et al. Comparison of placental elasticity in normal and pre-eclamptic pregnant women by acoustic radiation force impulse elastosonography. J Obstet Gynaecol Res 2016; 42: 1464-70. doi:10.1111/jog.13078

31. Ruscalzo A, Steinhard J, Pietro LA, Frohlich C, Bijnens B, Klockenbusch W, et al. Reliability of quantitative elastography of the uterine cervix in at-term pregnancies. J Perinat Med 2013; 41: 421-7. doi:10.1515/jpm-2012-0180 